\title{
Groucho proteins: transcriptional corepressors for specific subsets of DNA-binding transcription factors in vertebrates and invertebrates
}

\author{
Alfred L. Fisher and Michael Caudy ${ }^{1}$ \\ Department of Cell Biology and Anatomy, Cornell University Medical College, N ew York, N ew York 10021 USA
}

Extensive analysis during the last $10-15$ years has identified many of the mechanisms and factors involved in the activation of eukaryotic gene transcription. Although activation is better studied and more appreci ated, a growing body of work has shown that in many circumstances transcriptional repression is as important as activation in the regulation of gene expression (Gray and Levine 1996). Studies of the development of the Drosophila peripheral nervous system (PNS) have revealed that a family of basic helix-loop-helix (bHLH) transcriptional repressors, known as the Hairy-related proteins, plays critical roles during devel opment by repressing target genes at multiple stages of neurogenesis (Fisher and Caudy 1998). Similarly, the early patterning of the Drosophila embryo requires genes encoding transcriptional repressor proteins as well as transcriptional activator proteins, and mutations in either the activators or repressors result in lethal defects in patterning (Carroll 1990; Gray and Levine 1996). In mammals, the expression of certain neuron-specific genes like the type II sodium channel is restricted to neurons by the REST/ NRSF protein that transcriptionally represses these genes in the non-neuronal cells in which REST / NRSF is expressed (Chong et al. 1995; Schoenherr and Anderson 1995). In humans, the DAX-1 gene, which is mutated in congenital X-linked adrenal hypoplasia, encodes a transcriptional repressor, and mutations responsible for the disease phenotype map to its repression domain (Lalli et al. 1997; Zazopoulos et al. 1997). This review focuses on a family of transcriptional corepressor proteins, known as Groucho proteins, that are found in flies, mice, humans, frogs, and worms.

Groucho, the founding member of this family, was identified initially as a mutation that affects the development of the Drosophila nervous system, with one allele resulting in thick tufts of sensory bristles over the eyes, resembling the bushy eyebrows of the comedian Groucho M arx. The Groucho proteins serve as non-DN A binding corepressors for specific subsets of DNA-binding

${ }^{1}$ Corresponding author.

E-MAIL mcaudy@mail.med.comell.edu; FAX (212) 746-8175. transcription factors, including the Hairy-related proteins, Runt domain proteins, Engrailed, and Dorsal, and they are essential for certain aspects of repression by each of these repressors (Paroush et al. 1994; Fisher et al. 1996; A ronson et al 1997; Dubnicoff et al. 1997; Jimenez et al. 1997). These family members have been shown to be widely expressed both during development and in the adult, in contrast to the more limited expression pattern of their DNA-binding partners (Hartley et al. 1988; Stifani et al. 1992; Miyasaka et al. 1993; Schmidt and Sl adek 1993; Choudhury et al. 1997; Pflugrad et al. 1997; Sharief et al. 1997). In addition, both Groucho and the human family member TLE1 have been shown to actively repress transcription when fused to a heterol ogous DNA-binding domain and directly bound to DNA (Fisher et al. 1996). Hence, the Groucho proteins are recruited to target gene promoters by di rect binding to specific DNA-binding repressors, and once recruited, the Groucho proteins repress transcription via a conserved intrinsic repression domain.

\section{Groucho proteins act as corepressors for specific active repressors}

Groucho proteins are corepressors that are required for transcriptional repression by several distinct types of active transcriptional repressors. Corepressors are defined in this review as proteins that are required for the repressor activity of a specific transcription factor but do not have the ability to bind DNA alone. Hence, such corepressors are recruited to target promoters by proteinprotein interactions between a specific DNA-binding partner and the corepressor. For example, the mSin3 protein is a corepressor that is recruited to target promoters by the bHLH leucine-zipper repressor M ad as well as by unliganded nucl ear receptor proteins, such as the thyroid hormone receptor (Pazin and Kadonaga 1997). In addition, $\mathrm{mSin} 3$ has intrinsic repressor activity and represses target gene promoters when directly bound to DNA by a heterologous DNA-binding domain. In contrast to mSin3, the human Kap-1 protein serves as a corepressor for a specific subfamily of zinc-finger transcription fac- 
tors that contain a small domain known as the Krab domain to which Kap-1 binds (Friedman et al . 1996). The contrast between mSin3 and Kap-1 illustrates how some corepressors are relatively promiscuous and interact with several unrelated families of transcription factors, whereas others are more specialized and interact with a limited range of transcription factors. Finally, the $\mathrm{Rb}$ protein, which acts as a corepressor for the E2F transcription factor, shows another important property of at least some corepressors, that of regulation. The interaction between E2F and Rb is not constitutive but, instead, is regulated by phosphorylation of the $\mathrm{Rb}$ protein during the cell cycle (Sellers and Kaelin 1996). The ability of corepressor-DNA-binding partner interactions to be regulated allows some DNA-binding proteins to act as both repressors and activators, as is the case for E2F, and allows regulation by cell signaling or other pathways to modulate the control of target gene transcription.

The DNA-binding partners for the Groucho proteins all function as active transcriptional repressors in at least some contexts. Active repressors are distinguished from passive repressors by their mechanism of action (Cowell 1994). Passi ve repressors act by interfering with the transcriptional activator proteins that activate target gene transcription. This repression can occur by various mechanisms, such as competing for DN A sites bound by activator proteins, forming inactive heterodimers with activator proteins, or titrating coactivators required by the activator proteins (Cowell 1994). An example of a passive repressor is the Id protein, which represses transcription by forming non-DNA binding, inactive heterodimers with activator bHLH transcription factors such as M yoD (Benezra et al. 1990). In contrast, active repressors negatively regulate target genes by binding to repressor-specific sites in the target gene and repressing transcription by a distinct intrinsic repression domain (Cowell 1994). By this definition, many known transcripti onal repressor proteins are active repressors. Active repression domains are defined operationally as domains that are necessary for repression, and they can be found either in a DN A-binding protein or in a corepressor. Because of the modular nature of proteins, these domains often can also confer repression when fused to a heterol ogous DN A-binding domain. For example, zinc-finger proteins containing the Krab domain are active repressors because they bind to repressor-specific sites and repress via the recruitment of the Kap-1 corepressor (Friedman et al. 1996). In agreement with this, the Krab domain will repress transcription when fused to a heterologous DNA binding protein and targeted to that protein's binding sites. On the basis of these defining criteria, the various DNA-binding partners of the Groucho proteins can all act as active transcriptional repressors in at least some contexts (Jaynes and O'Farrel I 1991; Jiang et al. 1992; Pan and Courey 1992; Oellers et al. 1994 Ohsako et al. 1994; Van Doren et al . 1994; Aronson et al. 1997). In addition, the Groucho proteins al so act as active transcriptional repressors when directly bound to DN A by fusion to a heterologous DN A-binding domain, as discussed further bel ow (Fisher et al. 1996).

\section{Groucho proteins as corepressors for Hairy-related proteins, Runt domain proteins, Engrailed, and Dorsal in specific biological pathways}

\section{Hairy-related proteins}

The Hairy-related proteins are a family of bHLH transcription factors that, in Drosophila, are involved in segmentation, neurogenesis, sex determination, and myogenesis (for review, see Paroush et al. 1994; Fisher and Caudy 1998). In vertebrates, family members have been shown to be involved in neurogenesis and somite formation (Kageyama and Nakanishi 1997; Palmeirim et al. 1997). Additionally, members of this family such as the Drosophila Enhancer of spl it genes and certain mammalian Hairy/Enhancer of split (HES) genes are important effectors of the $\mathrm{N}$ otch signaling pathway, which controls neuronal cell fate decisions in both vertebrates and Drosophila (Kageyama and Nakanishi 1997; Robey 1997; Fisher and Caudy 1998). The mammalian HES-1 protein is also a functional target for N erve Growth Factor (N GF) signaling in the PC 12 cell line (Ström et al. 1997). In these various pathways, the Hairy-related proteins act genetically as repressors of target genes in vivo.

The Hairy-rel ated proteins are defined by the presence of two specific domains: a proline bHLH domain, which contains a proline at a specific position in the basic region, and a 4-amino-acid WRPW (Trp-Arg-Pro-Trp) domain found at the carboxyl terminus of the protein (Paroush et al. 1994; Fisher et al. 1996). As transcriptional repressors the Hairy-related proteins appear to act both by passive mechanisms, such as di rectly interacting with activator proteins (Sasai et al. 1992; Kageyama and N akanishi 1997), and al so by active mechanisms involving the binding to repressor specific DNA sites in target genes via the bHLH domain and the recruitment of the Groucho proteins (Sasai et al. 1992; Oellers et al. 1994; Ohsako et al. 1994; Paroush et al. 1994; Van Doren et al. 1994; Fisher et al. 1996; Heitzler et al. 1996; Kageyama and Nakanishi 1997).

The role of Groucho proteins in repression by the Hairy-related proteins has been studied by both biochemical and genetic means. Initially, a yeast two-hybrid screen performed with the Drosophila Hairy protein identified the Drosophila Groucho protein as a specific interacting protein (Paroush et al. 1994). Subsequently, both Groucho and the human TLE1 protein were shown to bind several Hairy-related proteins, and the WRPW motif was shown to be both necessary and sufficient for this interaction (Fig. 1) (Paroush et al. 1994; Fisher et al. 1996; Grbavec and Stifani 1996). The WRPW motif was initially proposed to act as a transcriptional repression domain for the Hairy-related proteins (Ohsako et al. 1994), and assays in cultured cells confirmed that this motif is a repression domain that by itself is sufficient to confer active transcriptional repression when fused to a heterologous DNA-binding domain (Fisher et al. 1996). The interaction between the WRPW motif and Groucho is required for transcriptional repression by these proteins both in Drosophila embryos and cultured cells (Par- 
Hairy-related Proteins

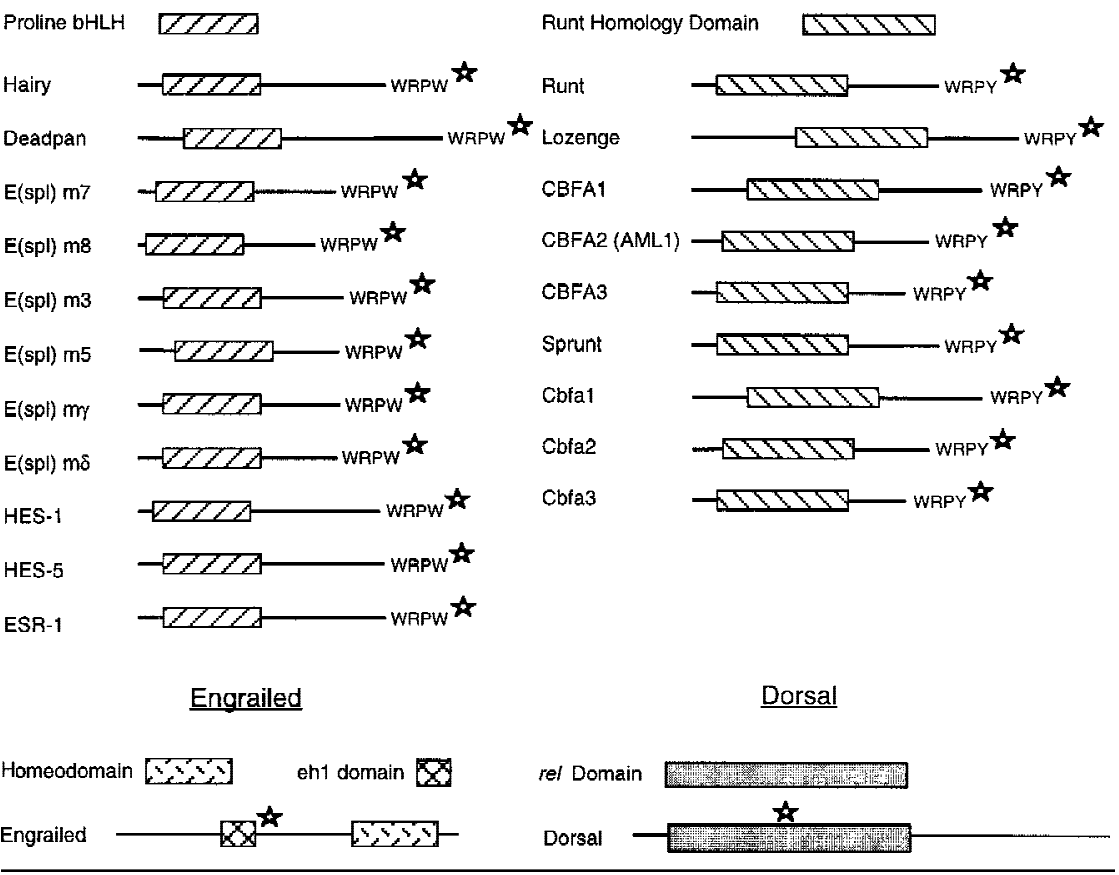

Figure 1. The DNA-binding partners of the Groucho proteins. (Rectangles) Different DN A-binding domains of the four families of transcription factors; (stars) interaction domains used by these proteins to interact with the Groucho proteins. oush et al. 1994; Fisher et al. 1996). Embryos lacking Groucho show defects in segmentation, neurogenesis, and sex determination that are phenotypes consistent with a functional role for Groucho as a corepressor for the Hairy-related proteins shown previously to be involved in these developmental processes (Paroush et al. 1994). This combination of in vitro biochemistry, transcriptional repression assays in cells, and Drosophila genetics indicated that the Groucho proteins are essential corepressors for the Hairy-rel ated proteins (Paroush et al. 1994; Fisher et al. 1996).

\section{Runt domain proteins}

The Runt domain family of transcription factors are found in both Drosophila and vertebrates. During Drosophila development, Runt and Lozenge play roles in segmentation, neurogenesis, sex-determination, and eye development (for review, see Duffy and Gergen 1994; Daga et al. 1996). In mammals, family members are essential for bone development and hematopoiesis (Speck and Terryl 1995; Okuda et al. 1996; Ducy et al. 1997; Komori et al. 1997; Otto et al. 1997; Rodan and Harada 1997). In humans, mutation or translocation of the AML1 and CBFA1 genes are a common occurrence in several forms of leukemia and lymphoma in adults and children, or are responsible for the inherited skeletal disorder cl ei docranial dysplasia, respectively (Lo Coco et al . 1997; Mundlos et al. 1997). The Runt domain is a distinct DNA-binding domain that mediates both DNA binding and heterodimerization with a non-DNA-binding partner, for example, Brother or Big-Brother in Drosophila or CBF $\beta$ in mammals (Speck and Terryl 1995).

Remarkably, at least one isoform of all Runt domain proteins ends with the sequence WRPY, which is very similar to the WRPW motif present in all Hairy-related proteins (Fig. 1; Aronson et al. 1997). The WRPY motif is both necessary and sufficient to mediate protein-protein interactions between the Drosophila Runt protein or the mouse homolog of the AML1 gene, PEBP $\alpha B 1$, and Groucho (Fig. 1; Alifragis et al. 1997; Aronson et al. 1997). Given the large number of Hairy-related proteins and Runt domain proteins currently identified in multiple species, it is quite striking that only Hairy-related proteins have the WRPW motif and only Runt domain proteins have the WRPY motif (Aronson et al. 1997). Why there is a strict division between these two transcription factor families and the four amino acid motifs used to interact with the Groucho proteins is currently unclear. Interestingly, the Runt domain proteins act as both transcriptional activators and repressors, whereas, in contrast, the Hairy-rel ated proteins appear to act only as transcriptional repressors (Aronson et al. 1997). One possible explanation is that Groucho interacts constitutively with the WRPW motif in Hairy-related proteins, whereas the interaction with WRPY -containing Runt domain proteins is regulated.

In Drosophila, the WRPY sequence and Groucho are essential for the repression of specific target genes by the Drosophila Runt protein (Aronson et al. 1997). A Runt protein lacking the WRPY motif is unable to repress transcription in cultured Drosophila cells or to repress the transcription of two specific target genes, hairy and even-skipped, in embryos (Aronson et al. 1997). However, this mutant still represses one in vivo target gene, engrailed, indicating that Runt also acts by an uncharacterized Groucho-independent repression mechanism (Aronson et al. 1997). In addition, the repression of the 
target genes hairy and even-skipped in embryos by Runt also depends on the level of Groucho protein in the embryo, which indicates that Groucho is necessary for Runt to repress target genes in vivo (Aronson et al. 1997). Additionally, the mammalian PEBP $\alpha B 1$ and AML1b proteins have al so been shown to have repressor activity in human HeLa cells, suggesting that Groucho-dependent transcriptional repression is a potential function of all Runt domain proteins with the WRPY motif (Aronson et al. 1997).

\section{Engrailed}

The Drosophila engrailed gene is a segment polarity gene that has roles in both the embryo and adult ( $M$ anak and Scott 1994). Engrailed is a homeobox protein (Fig. 1) that represses the transcription of target genes (Jaynes and O'Farrell 1991; Han and Manley 1993; Smith and Jaynes 1996). Recently, Engrailed has been shown to require Groucho for the repression of specific target genes in vivo (Jimenez et al. 1997). A repression domain from Engrailed located between amino acids 168 and 298 is composed of two subdomai ns (Jaynes and O'Farrell 1991; Han and Manley 1993; Smith and Jaynes 1996; Jimenez et al. 1997). The first domain, referred to as eh1 (Engrailed homology 1), is conserved in all Engrailed homologs and has been shown to be the primary repression domain in certain assays in transgenic flies (Smith and Jaynes 1996). The eh1 domain directly binds to Groucho (Fig. 1; Jimenez et al. 1997; Tolkunova et al. 1998); thus, the conservation of the eh1 domain in many other homeobox proteins from both Drosophila and vertebrates suggests that a subset of homeodomain transcriptional repressor proteins may show Groucho-dependent repression (Smith and Jaynes 1996; Jimenez et al. 1997). The second domain, referred to as region $D$, has been identified as the primary repression domain in transiently transfected cells (Han and Manley 1993). Similar to Runt, Engrailed appears to act by both Groucho-dependent and independent mechanisms during development because Engrailed still retains some residual repressor activity in flies when the ehl domain is deleted, and groucho mutant clones in the developing wing do not exhibit the same phenotype as engrailed mutant clones (de Celis and Ruiz-Gomez 1995; Smith and Jaynes 1996; Jimenez et al. 1997). This may be explained by the presence of both the eh1 domain and region $D$ within Engrailed as each subdomain could have independent repressor activity depending on the target gene being repressed. Hence, the eh1 domain/Groucho-mediated repression may only be requred for specific target genes, whereas other targets may require repression mediated by region $D$.

\section{Dorsal}

The Drosophila Dorsal protein also has been shown to interact biochemically with Groucho and to exhibit Groucho-dependent repression in vivo (Dubnicoff et al. 1997). Dorsal is a member of the NF-kB/rel family of transcription factors (Fig. 1) and during development is involved in specifying the dorsoventral axis of the Drosophila embryo (Courey and Huang 1995). In the ventral regions, Dorsal acts to activate the transcription of ventral-specific genes and simultaneously, in the same cells, to inhibit the transcription of dorsal-specific genes (Courey and Huang 1995). Dorsal has been shown to function intrinsically as a transcriptional activator that binds to sites in the promoters of the ventral-specific genes to activate their transcription (Courey and Huang 1995). Repression of the dorsal-specific genes requires the binding of Dorsal to sites within their promoters, and Groucho is al so required for Dorsal-mediated repression of these promoters (Jiang et al. 1992; Pan and Courey 1992; Courey and Huang 1995; Dubnicoff et al. 1997). Because the interaction between Dorsal and Groucho is mediated by the rel domain that is found in N F-кB and related mammalian transcription factors (Fig. 1; Dubnicoff et al. 1997), it will be important to determine whether Groucho proteins can also interact with these mammalian proteins to similarly repress specific target genes.

In summary, two findings indicate that the Groucho proteins function as active transcriptional corepressors for the various DNA-binding partners described above. First is the genetic requirement for Groucho for in vivo transcriptional repression by the various Drosophila partner proteins (Paroush et al. 1994; Aronson et al. 1997; Dubnicoff et al. 1997; Jimenez et al . 1997). Second is the observation that the Groucho proteins have intrinsic active transcriptional repressor activity when directly bound to target genes by fusion to a heterologous DN A binding protein (Fisher et al. 1996). Together with the biochemical interactions between Groucho proteins and the repression domains of these partner proteins, these two findings clearly indicate that the Groucho proteins are active transcriptional corepressors that are re cruited to target genes in vivo by specific subsets of DN A-binding proteins (Paroush et al. 1994; Fisher et al. 1996; A ronson et al. 1997; Dubni coff et al. 1997; Jimenez et al. 1997).

\section{Structural and functional domains of Groucho proteins}

The Groucho proteins from Drosophila, Caenorhabditis elegans, Xenopus, rats, mice, and humans all share a similar primary sequence structure consi sting of a series of seven highly conserved, carboxyl-terminal WD40 repeats (for discussion of WD40 repeats, see N eer et al. 1994), a highly conserved ami no terminus, and a variable region that separates these two domains (Fig. 2A,B) (Hartley et al. 1988; Stifani et al. 1992; Miyasaka et al. 1993; Schmidt and Sladek 1993; Choudhury et al. 1997; Pflugrad et al. 1997; Sharief et al . 1997). Proteins resembling the Groucho proteins, either consisting of part of the amino terminus but lacking the variable region and WD 40 repeats, or consisting of part of the variable region and the WD40 repeats, but lacking the amino terminus, have also been isolated (Fig. 2C; Stifani et al. 1992; M iyasaka et al. 1993; Schmidt and Sladek 1993; Choudhury et al. 1997). At this time we will not classify these as 
A

\begin{tabular}{c|c|c|}
\hline N-terminus & Variable & WD40 Repeats \\
\cline { 1 - 1 } $\begin{array}{c}\text { Transcriptional } \\
\text { Repression Domain } \\
\text { Dimerization }\end{array}$ & & Interaction with WRPW and WRPY \\
Interaction with EH1 Domain \\
Nucleą Localization? \\
Phosphoryiation?
\end{tabular}

B

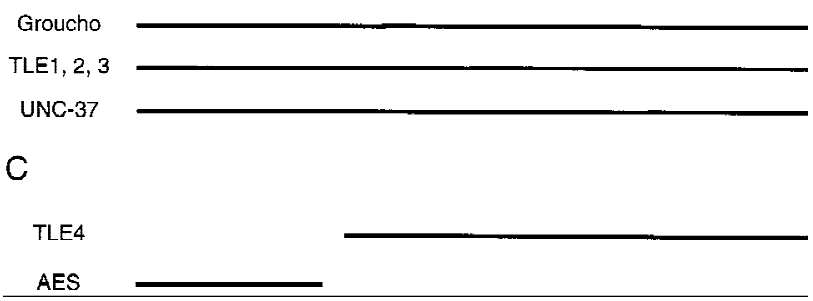

Figure 2. (A) Schematic diagram of the domain structure of Groucho proteins. Groucho proteins consist of three domains: the amino terminus, a series of seven carboxy-terminal WD40 repeats, and a variable region that separates the other two domains and shows poor sequence homology among family members. Below each domain are the functions that have been assigned to it. (B) Drosophila Groucho, human TLE 1, 2, and 3, and C. el egans UN C-37 proteins (solid lines) possess all three of these domains. Given the structural and functional similarities between these proteins, they are considered to be members of the Groucho protein family. (C) In contrast, the human TLE4 and mouse AES proteins lack some of these domains. Additionally, the biological function of these proteins is currently unknown, and they could act as dominant-negative inhibitors of the Groucho proteins. For these reasons, proteins such as these are considered here to be related to Groucho proteins, but not part of the Groucho protein family.

Groucho proteins because their function is not yet known. Although they may act as corepressors for some of the known partners or a distinct set of partners, these proteins could also act as antagonists of the Groucho proteins by titrating an effector or by binding to the targets of repression and acting as dominant-negative inhibitors.

The amino terminus of the Groucho proteins was shown to act as a dimerization and active repression domain (Fig. 2A; Fisher et al. 1996; Pinto and Lobe 1996). This domain also contains potential phosphorylation sites for cdc 2 and casein kinase II in close proximity to a nuclear localization sequence (Fig. 2A; Stifani et al. 1992). The amino terminus has been shown to repress both activated and basal transcription when bound directly to DNA and to have repressor activity equivalent to the full-length protein in several assays, suggesting that this domain is the major intrinsic repression domain (Fisher et al. 1996).

The exact mechanism used by the amino terminus to repress transcription is not known, but the recently described interaction between Groucho proteins and histone $\mathrm{H} 3$ may provide a mechanism, especially if the histone $\mathrm{H} 3$ interaction domain is found to localize to the amino terminus (Palaparti et al. 1997). An interaction with histone $\mathrm{H} 3$ could lead to the assembly or stabilization of repressive chromatin on target gene promoters and thereby repress transcription. This mechanism is used by the SIR repressors from yeast (Hecht et al. 1995), but for the Groucho proteins the role of the interaction with $\mathrm{H} 3$ in transcriptional repression is untested (Palaparti et al. 1997). The Groucho proteins may also work by other mechanisms in addition to, or instead of, the assembly of chromatin. Such mechanisms could include interaction with components of the basal transcription complex or the recruitment of other proteins with re pressor or enzymatic activity, such as the HDAC1 histone deacteylase protein that is known to interact with other transcriptional repressors (Pazin and Kadonaga 1997). Because the Groucho proteins can repress both activated and basal transcripti on (Fisher et al. 1996), they are unlikely to act via the quenching (Levine and M anley 1989) of the activation domains of transcriptional activators. Instead, the Groucho proteins appear to be general repressors that can repress both basal transcription and transcription activated by a variety of activator proteins.

On the basis of studies of other WD40 repeat proteins, the seven WD40 repeats found at the carboxyl terminus of the Groucho proteins (Fig. 2A) are likely to be involved in protein-protein interactions. WD 40 repeats generally function as protein-protein interaction domains and have been shown to form a $\beta$-propeller structure in which each repeat projects outward radially and is avail lable for interactions with other proteins ( $N$ eer et al. 1994; Sondek et al. 1996). For example, the WD40 repeats of the yeast TUP1 protein make direct contacts with the homeodomain protein al pha2 (Komachi et al. 1994; Komachi and Johnson 1997). The WD40 repeats of Groucho appear to be involved in making contact with Engrailed and Hairy as deletion of all of the repeats eliminates the interaction with these proteins (Jimenez et al. 1997). Additionally, the Groucho WD40 repeats were identified in a yeast two-hybrid screen as interacting with the eh1 domain of Engrailed (Tolkunova et al. 1998). However, the repeats al one are not sufficient to mediate a full interaction with either protein, so multi ple regions may be involved in the interaction (Jimenez et al. 1997). Further evidence for the importance of the WD40 repeats comes from recent work in C. el egans in which a Groucho protein, UN C-37, was shown to interact genetically with the homeodomain protein UNC-4 to control neuronal devel opment (Pflugrad et al. 1997). Several UN C-37 mutants have been sequenced, and most of the point mutations change specific amino acids within the WD 40 repeats (Pflugrad et al. 1997). Interestingly, a chimera containing the amino terminus of UN C-37 and the WD40 repeats of human TLE1 is able to rescue the UNC-37 phenotype, which suggests that the structure and function of the WD 40 repeats have been highly conserved among Groucho proteins (Pflugrad et al. 1997).

\section{Relationship of the Groucho proteins to the yeast corepressor TUP1}

The Groucho proteins have been compared with the 
yeast corepressor TUP1 because of the presence of carboxyl-terminal WD40 repeats in each protein and their common function as transcriptional corepressors. However, it is unclear whether these proteins represent true homologs as there are major structural and mechanistic differences between them. First, although the Groucho proteins and TU P1 both act as corepressors for members of multiple families of transcription factors, many of the DNA-binding partners for TUP1 do not directly interact with TUP1, but instead utilize an accessory protein known as CYC8 to form a stable complex (Kel eher et al. 1992; Tzamarias and Struhl 1995). In contrast to the Groucho proteins, which directly bind all currently studied partners, TUP1 di rectly binds only one DNA-binding partner, the $\alpha 2$ protein (Komachi et al. 1994; Komachi and Johnson 1997). Second, both TUP1 and the Groucho proteins have intrinsic repressor activity, and for TUP1 this activity appears to be mediated both by direct inhibition of the basal complex, perhaps via direct interaction with the RNA polymerase II-associated Srb proteins (Herschbach et al. 1994; Wahi and Johnson 1995; Carl son 1997; Redd et al. 1997), and by interaction with histones H3 and H4 (Edmondson et al. 1996). The TUP1 interaction with histones is mediated by a region that coincides with the identified TUP1 repression domain (Tzamarias and Struhl 1994; Edmondson et al. 1996). Whereas Groucho proteins also interact with histone H3 (Palaparti et al. 1997), and it is quite possible that direct interactions with histones are important for transcriptional repression by both proteins, no interactions of Groucho proteins with basal complex factors have yet been demonstrated. Third, the arrangement of functional domains and amino acid sequences of the proteins suggest that there may be significant differences. TUP1 does not conform to the general domain structure of the Groucho proteins discussed earlier as the immediate amino terminus of TUP1 is not required for transcriptional repression, but is instead involved in making contacts with the CYC8 accessory protein (Tzamarias and Struhl 1994). At the amino acid level, both the repression domains and WD40 repeats show poor sequence conservation, although it is possible that the three dimensional structures will show greater similarity. With these similarities and differences in mind, it may be more accurate to consider TUP1 and Groucho proteins as analogous rather than truly homologous.

\section{Activation vs. repression of transcription by Groucho partners}

Interestingly, some of the DN A-binding partners for the Groucho proteins do not always act as transcriptional repressors, and, in fact, some are better characterized as activators (Courey and Huang 1995; Speck and Terryl 1995). This is not unprecedented as other transcription factors that repress transcription with a corepressor also have been shown to activate transcription, including E2F and nuclear receptors such as the retinoic acid receptor (Sellers and Kael in 1996; Heinzel et al. 1997). Among the partners for the Groucho proteins, both Dorsal and the
Runt domain proteins are known to directly bind and activate the transcription of specific target genes (Courey and Huang 1995; Speck and Terryl 1995). For Dorsal and possibly the Runt domain proteins, the context of the target gene promoter appears to be critical for determining whether activation or repression will occur. Context here refers to the location and occupancy of DN A-binding sites for other proteins in the immediate vicinity of the binding site for a specific transcription factor. The context-dependent activities of these Groucho partner proteins suggest that the recruitment of Groucho proteins or their repressor activity might be inhibited in certain contexts. It al so is possi ble that Groucho proteins might even function as coactivators in certain situations.

The Drosophila Dorsal protein has been shown recently to bind directly to Groucho and to exhibit Groucho-dependent repression in vivo (Dubnicoff et al. 1997). Dorsal appears to repress transcription only when its binding sites are located near binding sites for DNAbinding proteins that have been termed corepressors (Fig. 3; Jiang et al. 1993; Kirov et al. 1993; Huang et al. 1995). Both Dorsal and corepressor binding sites are present in the promoters of the dorsal-specific genes, and these proteins act together with Groucho to repress the transcription of dorsal-specific genes in the ventral regions of the embryo. In contrast, ventral-specific genes lack corepressor sites and Dorsal activates transcription of these genes suggesting that Dorsal functions intrinsically as an activator of transcription (Fig. 3). One possible mechanism for dorsal-specific gene repression is that the corepressors al so directly bind to Groucho and thus stabilize a Dorsal-Groucho complex on DNA, and/or that the corepressors interact with Dorsal to enhance its ability to bind to Groucho (Fig. 3, double-headed arrows). Because Groucho and Dorsal can bind to each other in vitro in the absence of these DNA-binding corepressors (Dubnicoff et al . 1997) it will be important to determine whether the corepressors can potentiate these binding interactions.

The Runt domain proteins also both activate and repress target genes in a context-dependent manner. For example, the Drosophila Runt protein represses hairy and even-skipped expression, but activates the expression of fushi-tarazu and Sex-lethal (Duffy and Gergen 1994; Aronson et al. 1997). The mammalian Runt domain proteins have only been characterized as activators of a large number of target genes in blood cells and bone (Speck and Terryl 1995; Rodan and Harada 1997), although the recent demonstration of repressor activity for these proteins may lead to the identification of repressed targets (A ronson et al. 1997). However, in contrast to Dorsal, the Runt domain proteins appear to function intrinsically as transcriptional repressors, and to act only as activators in a context-dependent manner (Fig. 4; Speck and Terryl 1995; Aronson et al. 1997).

A well-studied example of transcriptional activation by the mammalian Runt domain proteins involves the $\mathrm{TCR} \alpha$ enhancer, which requires for full activation the simultaneous binding of the transcription factors CREB/ ATF, Ets, LEF-1, and the Runt domain protein AM L-1 to 
A: Context-Dependent Repression by Dorsal of Dorsal-Specific Genes

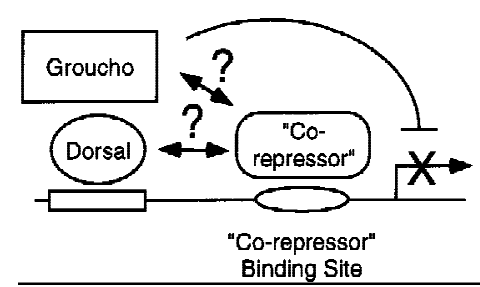

B: Intrinsic Activation by Dorsal of Ventral-Specific Genes

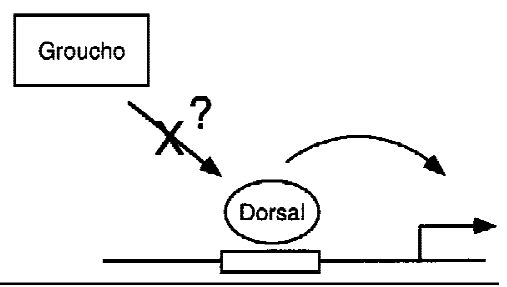

Figure 3. The Drosophila transcription factor Dorsal as a contex-dependent repressor and intrinsic activator of transcription depending on target gene promoter context. (A) Dorsal exhibits context-dependent repression of dorsal-specific genes such as zen, which contain Dorsal-binding sites along with binding sites for DNA-binding corepressor proteins. For Dorsal to act as a repressor, both Groucho and the corepressor proteins need to be present as removal of either Groucho or the binding sites for the corepressors results in activation instead of repression (see text). Dorsal has been shown to directly bind to Groucho, but whether there are protein-protein interactions (double-headed arrows) between Dorsal and the DNA-binding corepressors or between these corepressors and Groucho that help stabilize the DNA-bound Dorsal-Groucho complex remain important, unanswered questions. (B) When bound to ventralspecific genes such as twist that lack binding sites for the corepressors, Dorsal functions intrinsically as a transcriptional activator. specific sites (Fig. 4; Giese et al. 1995). The context dependence of this enhancer comes from at least two sources: the DNA bend produced by binding of the architectural protein LEF-1 and context-dependent activati on domains (CADs) present in AM L-1 and LEF-1 (C arlsson et al. 1993; Giese and Grosschedl 1993; Giese et al. 1995; Bruhn et al. 1997). For these two activation domains, transcriptional activation is only observed when the proteins containing them are bound to the TCR $\alpha$ enhancer at the correct location (Carlsson et al. 1993; Giese and Grosschedl 1993; Giese el al 1995). In contrast to most activators, no activation by these proteins is seen from artificial promoters with multimerized binding sites for AM L-1 or LEF-1 (C arlsson et al. 1993; Giese and Grosschedl 1993; Speck and Terryl 1995). Moreover, AM L-1 has been shown to bind di rectly to Groucho and to function intrinsically as a transcriptional repressor when fused to the heterologous GAL4 DN A-binding domain and bound to multimerized DNA-binding sites in an artificial promoter (Fig. 4B; Aronson et al. 1997).

The context-dependent activation domains in LEF-1 and AMLI bind a coactivator known as ALY that appears to stabilize DNA binding and facilitate transcriptional activation (Fig. 4; Bruhn et al. 1997). Additionally, AM L1 mutants lacking the WRPY motif that mediates interaction with the Groucho proteins still act as transcrip- tional activators, strongly suggesting that the Groucho proteins do not play a role in activation (Kurokawa et al. 1996). It will be interesting to determine whether, in contrast to Dorsal, the Runt domain proteins could intrinsically be repressors that are converted to activators through interactions with both coactivators and other transcription factors (Fig. 4). These interactions could either mask the WRPY motif, sterically block access of Groucho proteins to the Runt domain protein, or stabilize a conformation of the Runt domain protein that is unable to bind to Groucho proteins.

Thus, the Dorsal protein appears to be an intrinsic activator that can only repress transcription in the context of adjacent binding by specific cofactors. In contrast, the Runt domain protein AML-1 appears to be an intrinsic repressor that can only activate transcription in specific promoter contexts, perhaps in which the interaction with a Groucho protein is blocked by context-specific binding of cofactors. Additional studies will be needed to determine whether these are general properties for the various Runt domain and Rel domain proteins.

\section{Future directions}

Whereas significant progress has been made regarding
A: Context Dependent Activation by AML1 of the TCR $\alpha$ enhancer

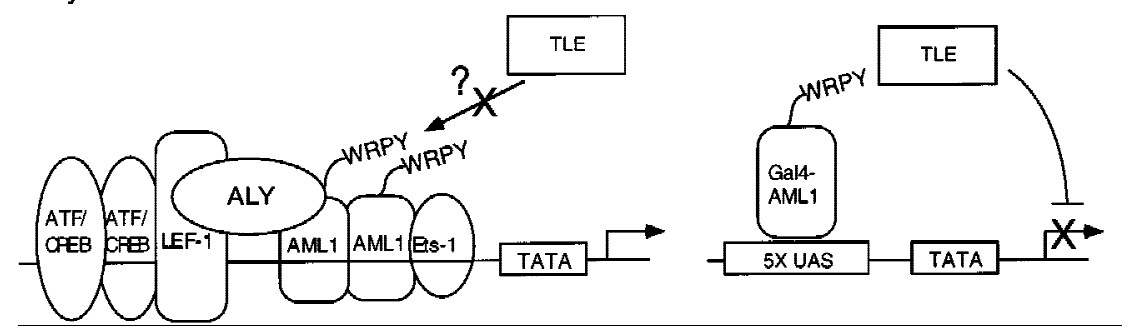

Figure 4. The mammalian AML1 protein as a context-dependent activator and an intrinsic repressor of transcription depending on target gene promoter context. (A) The TCR $\alpha$ enhancer is regulated by two transcription factors that are context dependent, including the Runt domain protein AML1. For complete activation of the enhancer, the transcription factors Ets, ATF/CREB, AML1, and LEF-1 must all assemble on the DNA, and AM L1 and LEF-1 need to interact with the coactivator ALY.

The binding of the HMG protein LEF-1 also induces a bend in the DNA (not shown here) that is important for assembly of the other factors on the enhancer. In contrast to the Ets and ATF/CREB proteins, AM L1 and LEF-1 will only activate transcription in the context of this promoter and only when their binding sites are located in the correct positions within the enhancer. An important question is how the AML1 protein acts as an activator of this enhancer when it has been shown to interact with Groucho and act as a transcriptional repressor when bound to an artificial promoter as a Gal 4 DN A-binding domain fusion protein. (B) Perhaps the assembly of these transcription factor proteins on the enhancer or the interaction with the coactivator ALY prevents an interaction between the WRPY motif of AML1 and Groucho proteins, thereby allowing context-dependent activation to occur. 
Groucho proteins and how they function, there are still many important questions that remain unanswered. First, the total number of families of DNA-binding partners for Groucho proteins is not known, and it is likely that there are more partners awaiting discovery. Second, the interaction of Groucho with transcription factors that both activate and repress transcription suggests that there must be regulation of the Groucho proteins at the level of either interaction with DN A-binding partners or their intrinsic repressor activity. A related question is whether Groucho proteins are regulated in more global ways, such as by cell signaling. There is preliminary evidence that cell signaling can regulate the Groucho proteins, as the phosphorylation status of several TLE proteins changes during the induction of neuronal differentiation of P19 cells by retinoic acid (Husain et al. 1996). Additionally, the Torso receptor tyrosine kinase inhibits the Groucho-dependent repressi on of tailless and huckebein expression in the anterior and posterior terminal regions of the Drosophila embryo. This regulation could occur either by inhibition of Groucho function or by inhibition of a yet to be characterized DNA-binding partner (Paroush et al. 1997). Third, an important mechanistic question is how the Groucho proteins repress both activated and basal transcription. The recent finding that Groucho proteins can interact with histone $\mathrm{H} 3$ may provide a mechanism, al though by analogy with TUP1 there may be multiple mechanisms involved in repression, including direct interactions with the basal transcriptional machinery.

\section{Acknowledgments}

We thank Drs. Claude Desplan, James Jaynes, Michael Levine, and Anthony M.C. Brown for helpful comments and discussion about this review. A.F. was supported in part by the Tri-Institutional M.D.-Ph.D. program [National Institutes of Health (NIH) Medical Science Training Program (MSTP) grant GM 07739] and funds from the Surdna Foundation. M.C. was supported by N IH grant R01 N S28652, the A Ifred P. Sloan Foundation, the Pew Scholars in Biomedical Sciences Program, and the Cornell Scholars Program.

\section{References}

Alifragis, P., G. Poortinga, S.M. Parkhurst, and C. Delidakis. 1997. A network of interacting transcriptional regulators involved in Drosophila neural fate specification revealed by the yeast two-hybrid system. Proc. Natl. Acad. Sci. 94: 13099-13104.

Aronson, B.D., A.L. Fisher, K. Blechman, M. Caudy, and J.P. Gergen. 1997. Groucho-dependent and -independent repression activities of Runt domain proteins. Mol. Cell. Biol. 17: 5581-5587.

Benezra, R., R.L. Davis, D. Lockshon, D.L. Turner, and H. Weintraub. 1990. The protein Id: A negative regulator of helixloop-helix DN A binding proteins. Cell 61: 49-59.

Bruhn, L., A. M unnerlyn, and R. Grosschedl. 1997. ALY, a context-dependent coactivator of LEF-1 and AM L-1, is required for TCR $\alpha$ enhancer function. Genes \& Dev. 11: 640-653.

Carlson, M. 1997. Genetics of transcriptional regulation in yeast: Connections to the RNA polymerase II CTD. Annu. Rev. Cell. Dev. Biol. 13: 1-23.
Carlsson, P., M.L. Waterman, and K.A. Jones. 1993. The hLEF/ TCF-1 $\alpha$ HMG protein contains a context-dependent transcriptional activation domain that induces the TCR $\alpha$ enhancer in T cells. Genes \& Dev. 7: 2418-2430.

Carroll, S.B. 1990. Zebra patterns in fly embryos: Activation of stripes or repression of interstripes? Cell 60: 9-16.

Chong, J.A., J. Tapia-Ramirez, S. Kim, J.J. Toledo-Aral, Y. Zheng, M.C. Boutros, Y.M. Altshuller, M.A. Frohman, S.D. Kraner, and G. Mandel. 1995. REST: A mammalian silencer protein that restricts sodium channel gene expression to neurons. Cell 80: 949-957.

Choudhury, B.K., J. Kim, H.F. Kung, and S.S. Li. 1997. Cloning and developmental expression of Xenopus CDNAs encoding the Enhancer of split groucho and related proteins. Gene 195: 41-48.

Courey, A.J. and J.D. Huang. 1995. The establishment and interpretation of transcription factor gradients in the Drosophila embryo. Biochim. Biophys. Acta 1261: 1-18.

Cowell, I.G. 1994. Repression versus activation in the control of gene transcription. Trends Biochem. Sci. 19: 38-42.

Daga, A., C.A. Karlovich, K. Dumstrei, and U. Banerjee. 1996. Patterning of cells in the Drosophila eye by Lozenge, which shares homologous domains with AML1. Genes \& Dev. 10: 1194-1205.

de Celis, J.F. and M. Ruiz-Gomez. 1995. groucho and hedgehog regulate engrail led expression in the anterior compartment of the Drosophila wing. Development 121: 3467-3476.

Dubnicoff, T., S.A. Valentine, G. Chen, T. Shi, J.A. Lengyel, Z. Paroush, and A.J. Courey. 1997. Conversion of dorsal from an activator to a repressor by the gl obal corepressor groucho. Genes \& Dev. 11: 2952-2957.

Ducy, P., R. Zhang, V. Geoffroy, A.L. Ridall, and G. Karsenty. 1997. Osf2/Cbfal: A transcriptional activator of osteoblast differentiation. Cell 89: 747-754.

Duffy, J.B. and J.P. Gergen. 1994. Sex, segments, and the central nervous system: Common genetic mechanisms of cell fate determination. Adv. Genet. 31: 1-28.

Edmondson, D.G., M.M. Smith, and S.Y. Roth. 1996. Repression domain of the yeast global repressor Tupl interacts directly with histones H3 and H4. Genes \& Dev. 10: 12471259.

Fisher, A.L. and M. Caudy. 1998. The function of Hairy-related bHLH proteins in cell fate decisions. BioEssays 20: 298-306.

Fisher, A.L., S. Ohsako, and M. Caudy. 1996. The WRPW motif of the hairy-related basic helix-loop-helix repressor proteins acts as a 4-amino-acid transcription repression and proteinprotein interaction domain. Mol. Cell. Biol. 16: 2670-2677.

Friedman, J.R., W.J. Fredericks, D.E. Jensen, D.W. Speicher, X.P. Huang, E.G. Neilson, and F.J. Rauscher III. 1996. KAP-1, a novel corepressor for the highly conserved KRAB repression domain. Genes \& Dev. 10: 2067-2078.

Giese, K. and R. Grosschedl. 1993. LEF-1 contains an activation domain that stimulates transcription only in a specific context of factor-binding sites. EMBO J. 12: 4667-4676.

Giese, K., C. Kingsley, J.R. Kirshner, and R. Grosschedl. 1995. Assembly and function of a TCR $\alpha$ enhancer complex is dependent on LEF-1-induced DNA bending and multiple protein-protein interactions. Genes \& Dev. 9: 995-1008.

Gray, S. and M. Levine. 1996. Transcriptional repression in development. Curr. Opin. Cell. Biol. 8: 358-364.

Grbavec, D. and S. Stifani . 1996. M olecular interaction between TLE1 and the carboxyl-terminal domain of HES-1 containing the WRPW motif. Biochem. Biophys. Res. Commun. 223: 701-705.

Han, K. and J.L. Manley. 1993. Functional domains of the Drosophila Engrailed protein. EMBO J. 12: 2723-2733. 
Hartley, D.A., A. Preiss, and S. Artavanis-Tsakonas. 1988. A deduced gene product from the Drosophila neurogenic locus, enhancer of split, shows homology to mammalian G-protein beta subunit. Cell 55: 785-795.

Hecht, A., T. Laroche, S. Strahl-Bolsinger, S.M. Gasser, and M. Grunstein. 1995. Histone $\mathrm{H} 3$ and $\mathrm{H} 4 \mathrm{~N}$-termini interact with SIR3 and SIR4 proteins: A molecular model for the formation of heterochromatin in yeast. Cell 80: 583-592.

Heinzel, T., R.M . Lavinsky, T.M. Mullen, M. Soderstrom, C.D. Laherty, J. Torchia, W.M. Yang, G. Brard, S.D. N go, J.R. Davie, E. Seto, R.N. Eisenman, D.W. Rose, C.K. Glass, and M.G. Rosenfeld. 1997. A complex containing N-CoR, mSin3 and histone deacetylase mediates transcriptional repression. Nature 387: 43-48.

Heitzler, P., M. Bourouis, L. Ruel, C. Carteret, and P. Simpson. 1996. Genes of the Enhancer of split and achaete-scute complexes are required for a regulatory loop between $\mathrm{N}$ otch and Delta during lateral signalling in Drosophila. Development 122: 161-171.

Herschbach, B.M., M.B. Arnaud, and A.D. Johnson. 1994. Transcriptional repression directed by the yeast al pha 2 protein in vitro. Nature 370: 309-311.

Huang, J.D., T. Dubnicoff, G.J. Liaw, Y. Bai, S.A. Valentine, J.M. Shirokawa, J.A. Lengyel, and A.J. Courey. 1995. Binding sites for transcription factor NTF-1/Elf-1 contribute to the ventral repression of decapentaplegic. Genes \& Dev. 9: 31773189.

Husain, J., R. Lo, D. Grbavec, and S. Stifani. 1996. Affinity for the nuclear compartment and expression during cell differentiation implicate phosphorylated Groucho/TLE1 forms of higher molecular mass in nuclear functions. Biochem. J. 317: 523-531.

Jaynes, J.B. and P.H. O'Farrell. 1991. Active repression of transcription by the engrailed homeodomain protein. EMBO J. 10: 1427-1433.

Jiang, J., C.A. Rushlow, Q. Zhou, S. Small, and M. Levine. 1992. Individual dorsal morphogen binding sites mediate activation and repression in the Drosophila embryo. EMBO J. 11: 3147-3154.

Jiang, J., H. Cai, Q. Zhou, and M. Levine. 1993. Conversion of a dorsal-dependent silencer into an enhancer: Evidence for dorsal corepressors. EMBO J. 12: 3201-3209.

Jimenez, G., Z. Paroush, and D. Ish-Horowicz. 1997. Groucho acts as a corepressor for a subset of negative regulators, including hairy and engrailed. Genes \& Dev. 11: 3072-3082.

Kageyama, R. and S. Nakanishi. 1997. Helix-loop-helix factors in growth and differentiation of the vertebrate nervous system. Curr. Opin. Genet. Dev. 7: 659-665.

Keleher, C.A., M.J. Redd, J. Schultz, M. Carlson, and A.D. Johnson. 1992. Ssn6-Tup1 is a general repressor of transcription in yeast. Cell 68: 709-719.

Kirov, N., L. Zhelnin, J. Shah, and C. Rushlow. 1993. Conversion of a silencer into an enhancer: Evidence for a co-repressor in dorsal-mediated repression in Drosophila. EMBO J. 12: 3193-3199.

Komachi, K. and A.D. Johnson. 1997. Residues in the WD repeats of Tup1 required for interaction with al pha2. Mol. Cell. Biol. 17: 6023-6028.

Komachi, K., M.J. Redd, and A.D. Johnson. 1994. The WD repeats of Tup1 interact with the homeo domain protein al pha 2. Genes \& Dev. 8: 2857-2867.

Komori, T., H. Yagi, S. N omura, A. Yamaguchi, K. Sasaki, K. Deguchi, Y. Shimizu, R.T. Bronson, Y.H. Gao, M. Inada, M. Sato, R. Okamoto, Y. Kitamura, S. Yoshiki, and T. Kishimoto. 1997. Targeted disruption of $\mathrm{Cbfal}$ results in a complete lack of bone formation owing to maturational arrest of osteoblasts. Cell 89: 755-764.

Kurokawa, M., T. Tanaka, K. Tanaka, S. Ogawa, K. Mitani, Y. Yazaki, and H. Hirai. 1996. Overexpression of the AML1 proto-oncoprotein in N IH 3T 3 cells leads to neoplastic transformation depending on the DNA-binding and transactivational potencies. Oncogene 12: 883-892.

Lalli, E., B. Bardoni, E. Zazopoul os, J.M. Wurtz, T.M. Strom, D. Moras, and P. Sassone-Corsi. 1997. A transcriptional silencing domain in DAX-1 whose mutation causes adrenal hypoplasia congenita. Mol. Endocrinol. 11: 1950-1960.

Levine, M. and J.L. Manley. 1989. Transcriptional repression of eukaryotic promoters. Cell 59: 405-408.

Lo Coco, F., S. Pisegna, and D. Diverio. 1997. The AML1 gene: A transcription factor involved in the pathogenesis of myel oid and lymphoid leukemias. Haematologica 82: 364-370.

Manak, J.R. and M.P. Scott. 1994. A class act: Conservation of homeodomain protein functions. Development (Suppl.) 6177.

Miyasaka, H., B.K. Choudhury, E.W. Hou, and S.S. Li. 1993. Molecular cloning and expression of mouse and human CDNA encoding AES and ESG proteins with strong similarity to Drosophila enhancer of split groucho protein. Eur. J. Biochem. 216: 343-352.

Mundlos, S., F. Otto, C. Mundlos, J.B. Mulliken, A.S. Aylsworth, S. Albright, D. Lindhout, W.G. Cole, W. Henn, J.H. Knoll, M.J. Owen, R. Mertelsmann, B.U. Zabel, and B.R. OIsen. 1997. Mutations involving the transcription factor CBFA1 cause cleidocranial dysplasia. Cell 89: 773-779.

N eer, E.J., C.J. Schmidt, R. N ambudripad, and T.F. Smith. 1994. The ancient regulatory-protein family of WD-repeat proteins. Nature 371: 297-300.

Oellers, N., M. Dehio, and E. Knust. 1994. bHLH proteins encoded by the Enhancer of split complex of Drosophila negatively interfere with transcriptional activation mediated by proneural genes. Mol. \& Gen. Genet. 244: 465-473.

Ohsako, S., J. Hyer, G. Panganiban, I. Oliver, and M. Caudy. 1994. Hairy function as a DN A-binding helix-loop-helix repressor of Drosophila sensory organ formation. Genes \& Dev. 8: 2743-2755.

Okuda, T., J. van Deursen, S.W. Hiebert, G. Grosveld, and J.R. Downing. 1996. AML1, the target of multiple chromosomal translocations in human leukemia, is essential for normal fetal liver hematopoiesis. Cell 84: 321-330.

Otto, F., A.P. Thornell, T. Crompton, A. Denzel, K.C. Gilmour, I.R. Rosewell, G.W. Stamp, R.S. Beddington, S. Mundlos, B.R. Olsen, P.B. Selby, and M.J. Owen. 1997. Cbfal, a candidate gene for cleidocranial dysplasia syndrome, is essential for osteoblast differentiation and bone development. Cell 89: 765-771.

Palaparti, A., A. Baratz, and S. Stifani. 1997. The Groucho/ Transducin-like enhancer of split transcriptional repressors interact with the genetically defined amino-terminal silencing domain of histone H3. J. Biol. Chem. 272: 26604-26610.

Palmeirim, I., D. Henrique, D. Ish-Horowicz, and O. Pourquie. 1997. Avian hairy gene expression identifies a molecular clock linked to vertebrate segmentation and somitogenesis. Cell 91: 639-648.

Pan, D. and A.J. Courey. 1992. The same dorsal binding site mediates both activation and repression in a context-dependent manner. EMBO J. 11: 1837-1842.

Paroush, Z., R.L. Finley, Jr., T. Kidd, S.M. Wainwright, P.W. Ingham, R. Brent, and D. Ish-Horowicz. 1994. Groucho is required for Drosophila neurogenesis, segmentation, and sex determination and interacts directly with hairy-related bHLH proteins. Cell 79: 805-815.

Paroush, Z., S.M. Wainwright, and D. Ish-Horowicz. 1997. 
Torso signalling regulates terminal patterning in Drosophila by antagonising Groucho-mediated repression. Development 124: 3827-3834.

Pazin, M.J. and J.T. Kadonaga. 1997. What's up and down with histone deacetylation and transcription? Cell 89: 325-328.

Pflugrad, A., J.Y. M eir, T.M. Barnes, and D.M. Miller. 1997. The Groucho-like transcription factor UNC-37 functions with the neural specificity gene unc-4 to govern motor neuron identity in C. el egans. Development 124: 1699-1709.

Pinto, M. and C.G. Lobe. 1996. Products of the grg (Grouchorelated gene) family can dimerize through the amino-terminal Q domain. J. Biol. Chem. 271: 33026-33031.

Redd, M.J., M.B. Arnaud, and A.D. Johnson. 1997. A complex composed of tupl and ssn 6 represses transcription in vitro. J. Biol. Chem. 272: 11193-11197.

Robey, E. 1997. N otch in vertebrates. Curr. Opin. Genet. Dev. 7: 551-557.

Rodan, G.A. and S. Harada. 1997. The missing bone. Cell 89: 677-680.

Sasai, Y., R. Kageyama, Y. Tagawa, R. Shigemoto, and S. Nakanishi. 1992. Two mammalian helix-loop-helix factors structurally related to Drosophila hairy and Enhancer of split. Genes \& Dev. 6: 2620-2634.

Schmidt, C.J. and T.E. Sladek. 1993. A rat homolog of the Drosophila enhancer of split (groucho) locus lacking WD-40 repeats. J. Biol. Chem. 268: 25681-25686.

Schoenherr, C.J. and D.J. Anderson. 1995. The neuron-restrictive silencer factor (NRSF): A coordinate repressor of multiple neuron-specific genes. Science 267: 1360-1363.

Sellers, W.R. and W.G. Kael in. 1996. RB as a modulator of transcription. Biochim. Biophys. Acta 1288: M 1-M5.

Sharief, F.S., S.C. Tsoi, and S.S. Li. 1997. CDN A cloning and genomic organization of enhancer of split groucho gene from nematode Caenorhabditis el egans. Biochem. Mol. Biol. Int. 43: 327-337.

Smith, S.T. and J.B. Jaynes. 1996. A conserved region of engrailed, shared among all en-, gsc-, N k1-, N k2- and msh-class homeoproteins, mediates active transcriptional repression in vivo. Development 122: 3141-3150.

Sondek, J., A. Bohm, D.G. Lambright, H.E. Hamm, and P.B. Sigler. 1996. Crystal structure of a G-protein beta gamma dimer at 2.1A resolution. Nature 379: 369-374.

Speck, N.A. and S. Terryl. 1995. A new transcription factor family associated with human leukemias. Crit. Rev. Eukaryot. Gene Expr. 5: 337-364.

Stifani, S., C.M. Blaumueller, N.J. Redhead, R.E. Hill, and S. Artavanis-Tsakonas. 1992. Human homologs of a Drosophila Enhancer of split gene product define a novel family of nuclear proteins. Nature Genet. 2: 119-127.

Ström, A., P. Castella, J. Rockwood, J. Wagner, and M. Caudy. 1997. Mediation of NGF signaling by post-translational inhibition of HES-1, a basic helix-loop-helix repressor of neuronal differentiation. Genes \& Dev. 11: 3168-3181.

Tolkunova, E.N., M. Fujioka, M. Kobayashi, D. Deka, and J.B. Jaynes. 1998. Two distinct types of repression domain in Engrailed: One interacts with the Groucho corepressor and is preferentially active on integrated target genes. Mol. Cell. Biol. 18: 2804-2814.

Tzamarias, D. and K. Struhl. 1994. Functional dissection of the yeast Cyc8-Tup1 transcriptional co- repressor complex. Nature 369: 758-761.

- - 1995. Distinct TPR motifs of Cyc8 are involved in re cruiting the Cyc8-Tup1 corepressor complex to differentially regulated promoters. Genes \& Dev. 9: 821-831.

Van Doren, M., A.M. Bailey, J. Esnayra, K. Ede, and J.W. Posakony. 1994. N egative regulation of proneural gene activity:
Hairy is a direct transcriptional repressor of achaete. Genes \& Dev. 8: 2729-2742.

Wahi, M. and A.D. Johnson. 1995. Identification of genes required for alpha 2 repression in Saccharomyces cerevisiae. Genetics 140: 79-90.

Zazopoulos, E., E. Lalli, D.M. Stocco, and P. Sassone-Corsi. 1997. DN A binding and transcriptional repression by DAX-1 blocks steroidogenesis. Nature 390: 311-315. 


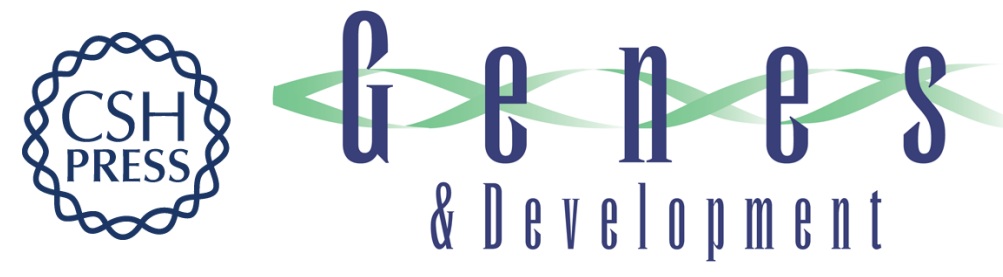

\section{Groucho proteins: transcriptional corepressors for specific subsets of DNA-binding transcription factors in vertebrates and invertebrates}

Alfred L. Fisher and Michael Caudy

Genes Dev. 1998, 12:

Access the most recent version at doi:10.1101/gad.12.13.1931

References

This article cites 79 articles, 32 of which can be accessed free at: http://genesdev.cshlp.org/content/12/13/1931.full.html\#ref-list-1

License

Email Alerting

Receive free email alerts when new articles cite this article - sign up in the box at the top Service right corner of the article or click here.

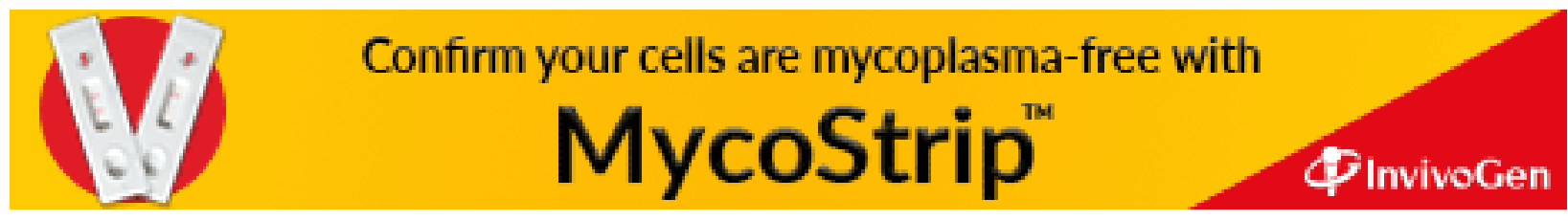

